\title{
A Case Study on the Relationship between Personality Traits and Parameters of Social Networks
}

\author{
Xiaowei Yan', Junhui Gao² \\ ${ }^{1}$ Jiangsu Tianyi High School, Wuxi, China \\ ${ }^{2}$ American and European International Study Center, Wuxi, China \\ Email: Jhgao68@163.com
}

Received 9 June 2016; accepted 18 July 2016; published 25 July 2016

\begin{abstract}
In this paper, the relationship between personality traits and the parameters of certain social networks is investigated directly through data collection by precise questionnaire surveys. First, social network analysis is employed to calculate multiple parameters of the networks. Then, a correlation analysis is conducted on the relationship between the personality traits and the parameters. The results show that some network parameters are in consistent with the students' personality traits. Results also reveal that the parameters of the respondents who mark themselves as "extroverted" are much closer to those of respondents who mark themselves as "neutral" than "introverted".
\end{abstract}

\section{Keywords}

Personality Traits, Social Network Analysis, Correlation Analysis

\section{Introduction}

There are three traits which comprise human personality: extraversion-introversion, neuroticism, and psychoticism. They are manifested in daily interpersonal communication. Social network analysis [1], developed by sociologists, is a quantitative analysis method based on mathematical methods and graph theories. During recent years, the method has played an important role and is widely used in the field of occupational mobility, the impact of urbanization on individual happiness, world political and economic system, international trade, etc. Social network analysis, a relatively mature field of sociology analysis, can be used by sociologists to interpret some of the sociological problems more handily. Social network analysis is one of the most significant research methods experts would adopt when they confront challenges.

Sheman (2000) used social network analysis method to analyze the relationship among the students of a class [2].

Wen Yue Zhu, Jun Hui Gao (2014) use social network analysis to analyze the inner relationship within a designated class. They employ Cytoscape software to calculate parameters of each student in the network including 
Degree, EcCentricity, Closeness and Betweenness. By using social network analysis, they try to identify the most popular, least popular and most bizarre characteristics of the students. The results later proved to be consistent with the one given by the teacher. The paper further carried out a preliminary analysis on the mechanism of news spreading between the male and female students [3] [4].

In the references [2]-[4], the relationship between the students is discussed based upon "three favorite classmates," "three least favorite classmates" listed by respondents. We also investigated the "three most frequently contact students", using NetDraw software to calculate a variety of network parameters. We also investigated the students' own personality traits data into outgoing, introverted and neutral. We try to figure out the relationship between the network parameters and the personality traits.

\section{Data Collection}

Questionnaires are used. Each student is asked to fill in their name on the questionnaire, the gender, the most contact with the contact name and three classmates least three students and a preliminary analysis (outward, inward, neutral) own character. Investigators would ensure the confidentiality of the survey results to the students in advance, in order to ensure that students fill out the authenticity of the information.

Data will be collected by sorting. Vertical columns in the order marked the students' exposure to and contact with the most minimal of students to learn numbers in the corresponding horizontal.

\section{Data Analysis}

\subsection{Computations of Network Parameters}

The survey data import software NetDraw [5], various network parameters can be calculated. Since the "greatest exposure” is directional, so we chose at the time of the analysis “digraph". Network parameters are obtained Degree, Betweeness, Closeness, Harmonic Closeness, Eigenvector like [6]. Next, some of these parameters will be described.

Degree, degree vertices, the number refers to the vertex associated with the edges. In a directed graph, there InDegree and OutDegree. InDegree expressed here by other students that the most contact with the number, the greater the value InDegree, indicating that the greater the degree of students welcome. InDegree maximum students can be considered the best class in popularity. OutDegree, all students should all be 3 .

BetweennessCentrality, referred Betweenness, betweenness centrality. Betweenness concept stems from the importance of social networks in the Individual. Simply put, Betweenness between a node represents the number of all the nodes on the shortest path of that node. Betweenness a good description of a network node may need to carry. Betweenness a node of the larger data packets passing through it, the more congestion means that it is easier to become a network bottleneck. Betweenness used to characterize the nodes in the network to influence the dissemination of information to measure the ability of a person as a medium. Consider a directed graph, where the calculated is BetweenessDir.

Tightness Closeness, vertex, metric to consider is the concept of the center, by the distance between the vertices of each figure to measure. Tightness vertex greater, indicating the apex living in the center of the network, the more important it in the network. Consider a directed graph, where the calculated and is In Closeness Out Closeness. Harmonic Closeness is a similar index and Closeness.

Eigenvector Centrality, referred Eigenvector, eigenvector centrality, a network node is a measure of importance. Each network node has a relative index value, which is based on the principle-the contribution of the high index node connected to a node node index lower than the contribution of high. Google's PageRank is a variant of the eigenvector centrality measure. eigenvector those other actors with specific actors is linked into account the centrality of an actor and a measure of central indicators. For example, a friend, there are many links its three target actors, with another three friends which have no link actors compared to the former"s higher center of feature vectors.

Table 1 lists some of the students of a class of five network parameters: InDegree, OutDegree, BetweenessDir, InCloseness, OutCloseness.

\subsection{Data Analysis of Disposition}

Analyze the disposition data of A, B two classes. Statistical results are shown in Table 2. From Table 2, we can 
Table 1. Part of the network parameters of seven students in a class.

\begin{tabular}{cccccc}
\hline \multirow{2}{*}{ Student ID } & \multicolumn{5}{c}{ Network parameters } \\
\cline { 2 - 6 } & InDegree & OutDegre & BetweenessDir & InCloseness & OutCloseness \\
\hline$\ldots$. & 3 & & & & 225 \\
11 & 3 & 2 & 89.21 & 228 & 204 \\
12 & 1 & 3 & 119.21 & 206 & 192 \\
13 & 5 & 3 & 34.03 & 193 & 197 \\
14 & 3 & 3 & 163.53 & 176 & 179 \\
15 & 4 & 5 & 108.48 & 179 & 176 \\
16 & 5 & 3 & 156.19 & & 1157 \\
\end{tabular}

Table 2. Data analysis of disposition in two classes.

\begin{tabular}{cccc}
\hline \multirow{2}{*}{ Class } & \multicolumn{2}{c}{ Disposition } \\
\cline { 2 - 4 } & Outgoing & Neutral & Introverted \\
\hline Class A & 12 & 18 & 5 \\
Class B & 8 & 24 & 4 \\
\hline
\end{tabular}

see that, A, B two classes of students they think are neutral character of the majority, more than 50\%. Followed by the outgoing, and finally introverted.

\subsection{Correlation Analysis between Network Parameters and Disposition}

We use SQL technology, computing network parameters mean A, B two classes for all students, and their correspondence with the character parameters, and the results are shown in Table 3.

Data in Table 3 show, InDegree average of the maximum is neutral character of students, not extroverted students. We mean the relationship BetweenessDir and outgoing, neutral, Private histogram is represented as shown in Figure 1. We found that the average parameters BetweenessDir gradual decline by extroversion, neutral, inward direction.

The relationship InCloseness and outgoing, neutral, Private histogram is represented as shown in Figure 2. We found that the average value of the parameter InCloseness press outward, neutral, inward direction is escalating.

We mean the relationship InEigenVector and outgoing, neutral, Private histogram is represented as shown in Figure 3. We found that the average value of the parameter InEigenVector press outward, neutral, inward direction is a gradual decline.

We found from Figure 2, Figure 3, the value of network parameters, the neutral value Social value is less than the distance value within the neutral value. On the facts, Table 3 also has another argument InHarmonicCloseness this tendency.

\subsection{Correlation Analysis between the Social Structure and the Disposition}

About the only definition of a network of community structure has not yet been widely recognized, more commonly used is based on the definition of the relative frequency of connections: a network of vertices can be divided into groups, the group connection between dense and sparse group connection.

In NetDraw software, data A class using Girvan-Newman algorithm [7] to calculate the number of modules of different societies (Q-value), divided into three associations found, the highest module. Factions calculated 


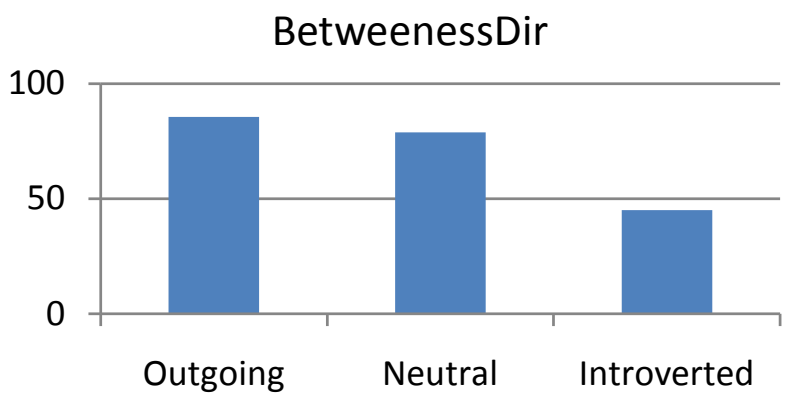

Figure 1. The relationship between BetweenessDir and three characters.

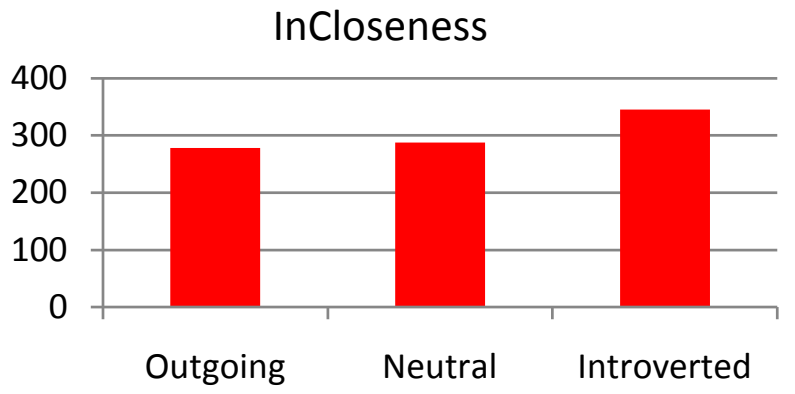

Figure 2. The relationship between InCloseness and three characters.

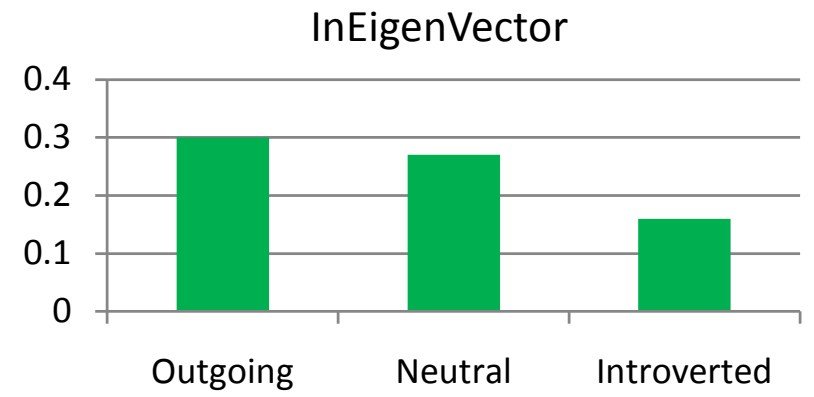

Figure 3. The relationship between InEigenVector and three characters.

Table 3. Correlation analysis between network parameters and disposition.

\begin{tabular}{cccc}
\hline \multirow{2}{*}{ Network Parameters } & & Disposition & Introverted \\
\cline { 2 - 4 } & Outgoing & Neutral & 2.44 \\
InDegree & 3.10 & 3.19 & 45.00 \\
BetweenessDir & 85.48 & 78.80 & 345.22 \\
InCloseness & 278.35 & 288.14 & 0.16 \\
InEigenVector & 0.30 & 0.27 & 10.35 \\
InHarmonicCloseness & 11.74 & 11.51 & \\
\hline
\end{tabular}

using the "most contact" network of relationships in the community structure or group, choose the number of packets of 3 results shown in Figure 4.

It can be found under the Societies aggregation nodes analysis results with different colors of different groups of nodes.

We made these students group information and the survey would like to combine the disposition data, the results of Table 4. From Table 4, we can see that there is no one group within the student; maximum number of 


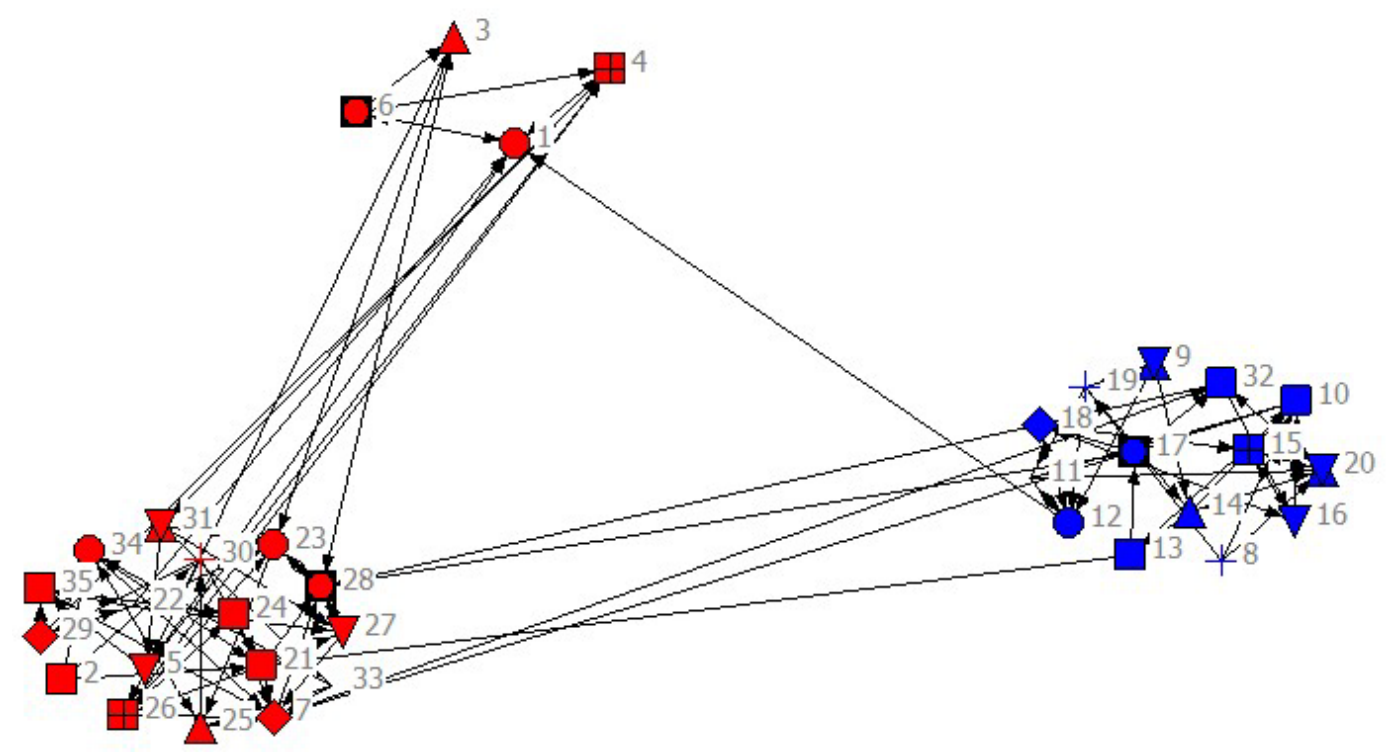

Figure 4. The subgroups of class A.

Table 4. The subgroups of class A and their disposition.

\begin{tabular}{cccc}
\hline & & Disposition & Introverted \\
\cline { 2 - 4 } Group & Outgoing & Neutral & 0 \\
Group 1 & 1 & 3 & 3 \\
Group 2 & 5 & 6 & 1 \\
Group 3 & 6 & & 9 \\
\hline
\end{tabular}

group 2, wherein the neutral character who account for more than half; group 3 and neutral to Chinese and foreign students are 6 people.

\section{Conclusion}

In this paper, social network analysis is employed to investigate the relationship between the traits of the network of classmates and the personality traits of these students. First, questionnaires are distributed to these respondents. By asking the respondents to fill out their personal information and the fellow students with whom they have "the most frequent contact", we get preliminary information about the network of the classmates within two classes in a local middle school and their personality traits data. Then, NetDraw software is employed to calculate multiple parameters of the class network. Mysql software is also used to process the data. At last; we found that the group of respondents who have the highest mean value of InDegree are students who have a neutral character instead of a extroverted one. Network parameters such as BetweenessDir, InCloseness, InEigenVector, InHarmonic Closeness are found out to be in consistent with the students' personality traits. In addition, we conduct a community structural analysis on the network of "the most frequent contact" and a correlation analysis on the information of the community and the personality traits.

\section{Acknowledgements}

The author thanks the mother of Xiaowei Yan for her significant contribution for collecting data.

\section{References}

[1] MBALib. http://wiki.mbalib.com/wiki/Social_network_analysis 
[2] Sheman, L. (2000) Sociometry in the Classroom: How to Do It. http://www.users.muohio.edu/shermalw

[3] Zhu, W.Y. and Gao, J.H. (2014) A Case Study on the Relationship between Classmates Based on Social Network Analysis. Science and Technology Innovation Herald, 29, 2014.

[4] Zhu, W.Y. and Gao, J.H. (2015) Analysis and Result Confirmation of Cohesive Subgroup Structure among Classmates. International Conference on Industrial Technology and Management Science (ITMS 2015). http://dx.doi.org/10.2991/itms-15.2015.180

[5] NetDraw. http://www.analytictech.com/downloadnd.htm

[6] van Steen, M. (2010) Graph Theory and Complex Networks: An Introduction. Maarten van Steen. 5 April 2010.

[7] Girvan, M. and Newman, M.E.J. (2002) Community Structure in Social and Biological Networks. Proc. Natl. Acad. Sci. USA, 99, 7821-7826. http://dx.doi.org/10.1073/pnas.122653799

\section{Submit or recommend next manuscript to SCIRP and we will provide best service for you:}

Accepting pre-submission inquiries through Email, Facebook, Linkedin, Twitter, etc A wide selection of journals (inclusive of 9 subjects, more than 200 journals)

Providing a 24-hour high-quality service

User-friendly online submission system

Fair and swift peer-review system

Efficient typesetting and proofreading procedure

Display of the result of downloads and visits, as well as the number of cited articles

Maximum dissemination of your research work

Submit your manuscript at: http://papersubmission.scirp.org/ 